\title{
Chemical Enrichment of the First Binaries
}

\author{
Ke-Jung Chen ${ }^{1,2, *}$ \\ ${ }^{1}$ Division of Theoretical Astronomy, National Astronomical Observatory of Japan, \\ Tokyo, Japan \\ ${ }^{2}$ Department of Astronomy \& Astrophysics, University of California, \\ Santa Cruz, California, USA \\ *EACOA Fellow, email: ken.chen@nao.ac.jp
}

\begin{abstract}
Recent studies of Population III (Pop III) star formation suggest that the primordial star-forming clouds can fragment to form binary or multiple stellar systems inside dark matter minihalos. Here, we briefly present the chemical enrichment of the Pop III binary scenarios.
\end{abstract}

Keywords. stars: formation - stars: Population III - galaxies: formation - early universe.

\section{Summary}

Understanding the first galaxy formation at the end of the cosmic dark ages is one of the grandest problems in modern cosmology. This formation process is significantly affected by the chemical enrichment from the Pop III supernovae (SNe) formed earlier in the minihalo progenitor systems. Recent cosmological simulations with extremely high resolution have shown that binary systems may be the typical channel for primordial star formation in minihalos. Since the evolution of binary systems and their final fate are very different from those of single stars, it is worthwhile to investigate whether binary stars lead to significantly altered SN feedback. Chen et al. (2015) studied the SN feedback of Pop III binary and single stars. They suggest that Pop III single stars are likely to die as black holes (BHs), or possibly accompanied by very weak SN explosions. A weak SN only carries an explosion energy of $\sim 0.1 \mathrm{~B}$ and a metal yield of $\sim 0.1 M_{\odot}$, due to strong fallback during the $\mathrm{BH}$-forming explosion. However, the enrichment from the binary systems can be more robust, because they contain less massive stars which are easier to blow up as core-collapse SNe. Thus, the recent revision of the Pop III star formation paradigm away from a single-star outcome to ubiquitous binarity in effect enhances chemical feedback in the early Universe.

\section{Acknowledgements}

K.C. acknowledges the support of EACOA Fellowship from the East Asian Core Observatories Association. Work at UCSC has been supported by an IAU-Gruber Fellowship, the DOE HEP Program (DE-SC0010676) and the NASA Theory Program (NNX14AH34G). Numerical simulations are supported by the Minnesota Supercomputing Institute (MSI), the National Energy Research Scientific Computing Center (NERSC), and the Center for Computational Astrophysics (CfCA) at National Astronomical Observatory of Japan (NAOJ).

\section{Reference}

Chen, K.-J., Bromm, V., Heger, A., Jeon, M., \& Woosley, S. 2015, ApJ, 802, 13 